\title{
Outcomes of Epilepsy Surgery in Drug Resistant Epileptic Patients in Loghman Hakim Hospital
}

\author{
Mahmoud Lotfinia ${ }^{1,2,3}$, Ahmad Ali Lotfinia ${ }^{3}$, Babak Khodaie ${ }^{3}$, Guive Sharifi ${ }^{*}$ \\ ${ }^{1}$ Clinical Research Development Center, Loghman Hakim Hospital, Shahid Beheshti University of Medical Sciences, Tehran, Iran \\ ${ }^{2}$ School of Medicine, Shahid Beheshti University of Medical Sciences, Tehran, Iran \\ ${ }^{3}$ Shefa Neuroscience Research Center, Khatam Alanbia Hospital, Tehran, Iran
}

\section{ABSTRACT}

Introduction: Drug-resistant epilepsy surgery has countless biological and psychological complications. Many of these patients can be treated with epilepsy surgery. Despite numerous surgeries in Iranian medical centers, only the results of few of them are published. Materials and Methods: To assess the outcome of the surgery, a total number of forty patients who undergone operation between 2004 and 2014 in Loghman Hakim hospital are included in our retrospective study. Results: $52.5 \%$ of operated patients, who are included in first class of Engel criteria, were completely free of seizures. The second, third and fourth class of Engel criteria contain 25, 15 and 7.5 percent of the operated patients. There was no statistically significant difference in surgery outcomes based on the gender, primary seizure type, and surgery approach. Only 5\% of the patients have permanent neurological complications, most commonly hemiparesis. Conclusion: The results of this study show the importance of early diagnosis and regular monitoring of epileptic patients. This significantly decreases the rate of unnecessary drug intake and increases the number of symptom-free individuals.

\section{Key words:}

1. Epilepsy

2. General Surgery

3. Drug Resistant Epilepsy

*Corresponding Author: Guive Sharifi

E-mail:gibnow@yahoo.com

doi: 10.18869/acadpub.shefa.5.1.48 
نتايج جراحى صرع در بيماران مبتلا به صرع مقاوم به دارو در بيمارستان لقمان حكيم

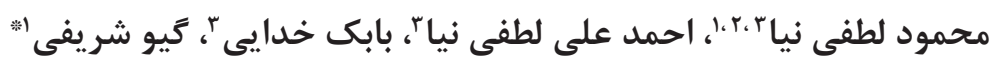 \\ 'مركز توسعه تحقيقات بالينى، بيمارستان لقمان حكيم، دانشكاه علوم يزشكى شهيد بهشتى، تهران، ايران

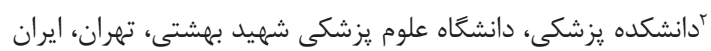

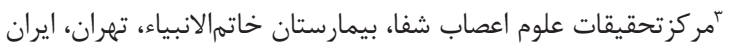

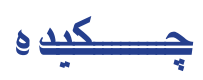

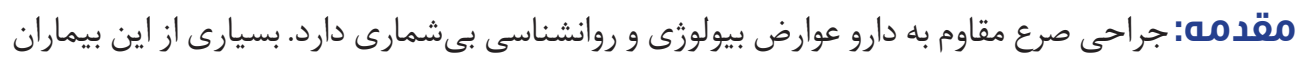

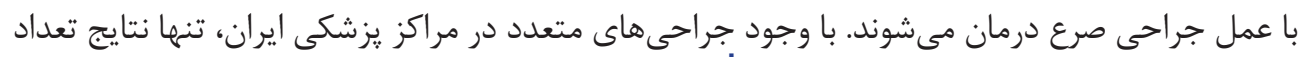

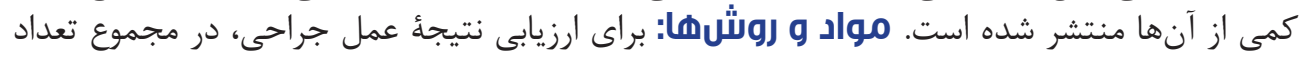

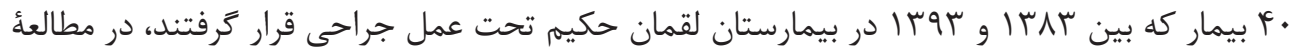

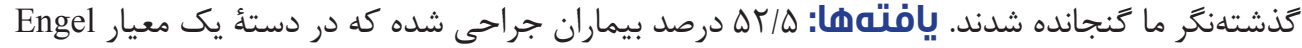

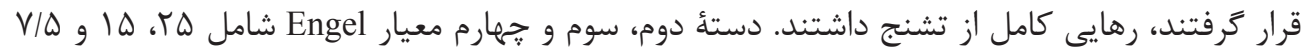

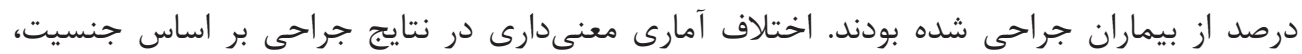

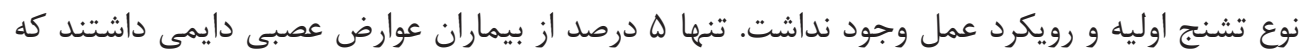

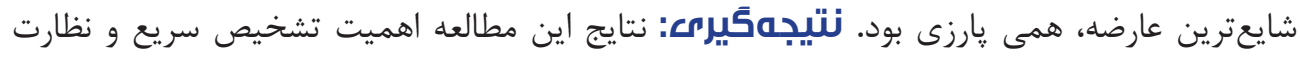

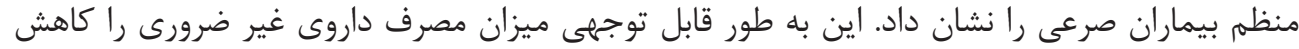
مىدهد و تعداد افراد بدون علامت ران را افزايش مي بـدهد.

\footnotetext{
كليد وازهها: I

r. جراحسى عمومسىى

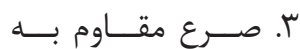
درمـان
} 


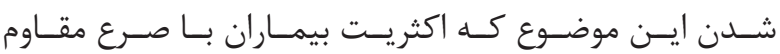

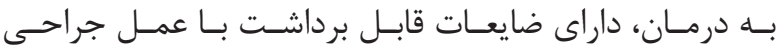

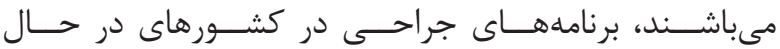

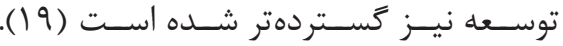

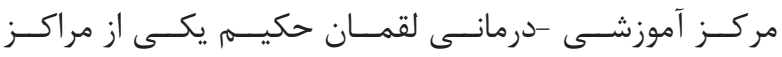

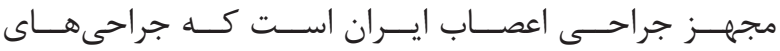

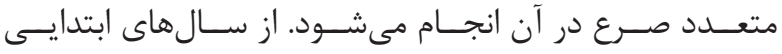

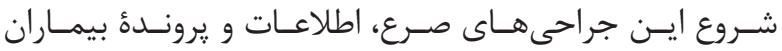

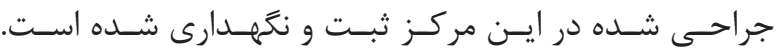

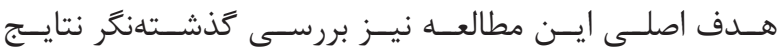

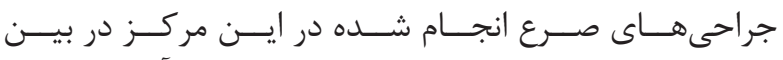

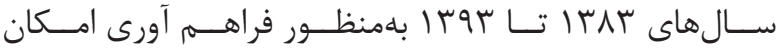

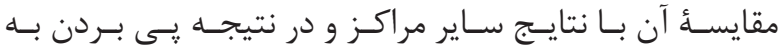

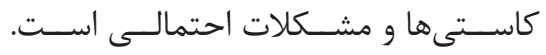

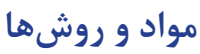

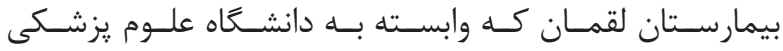

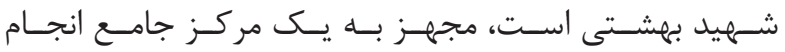

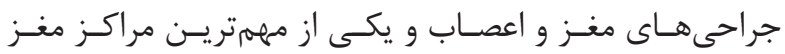

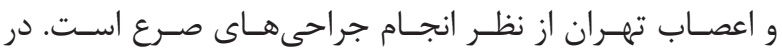

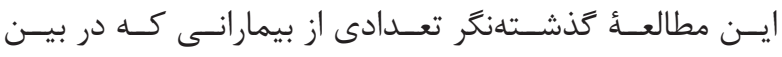

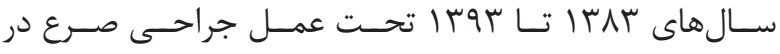

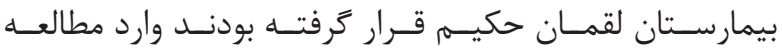

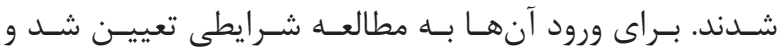

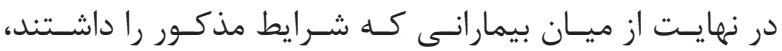

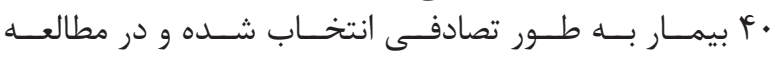

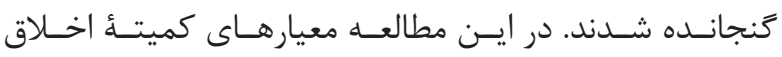

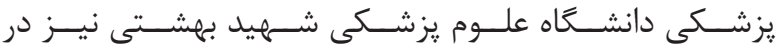

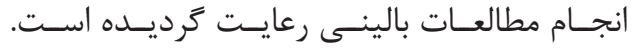

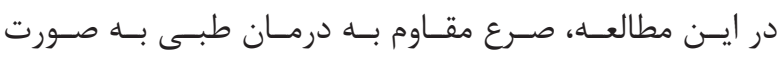

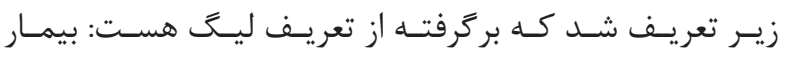

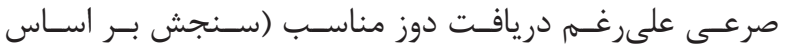

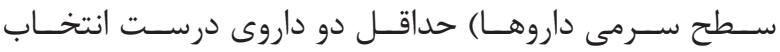

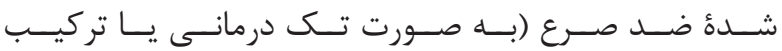

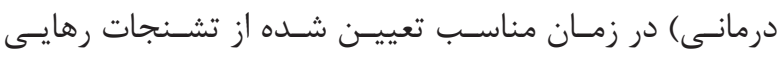

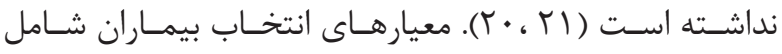

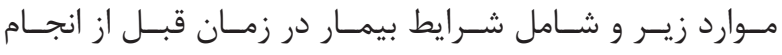

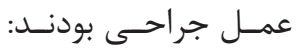

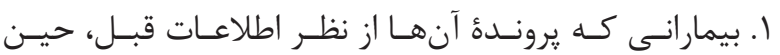

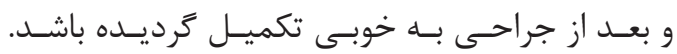

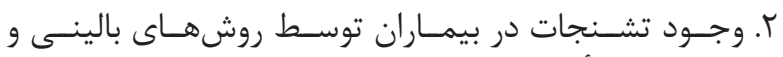

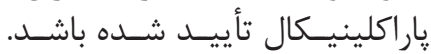

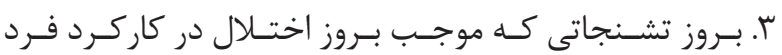

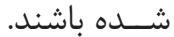
†. ب. بروز تشنجهاى مقاوم به درمان طبى.

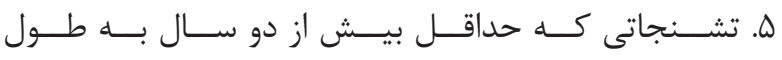

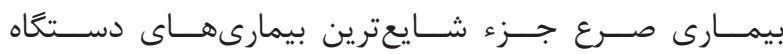

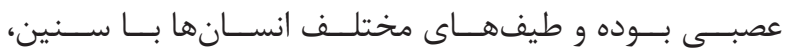

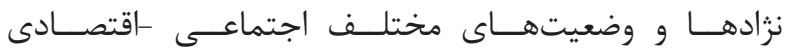

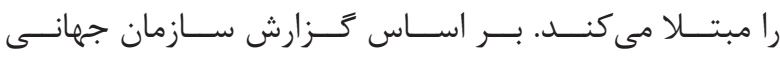

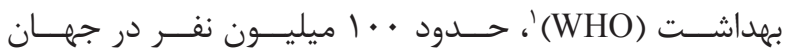

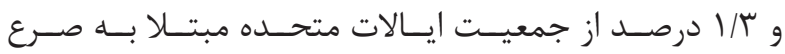

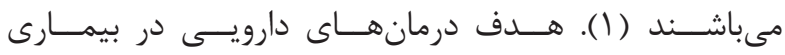

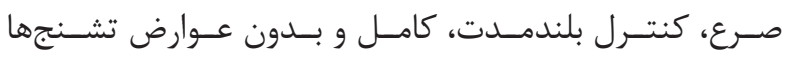

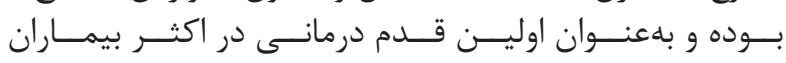

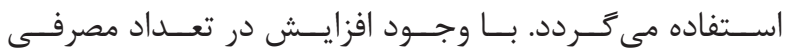

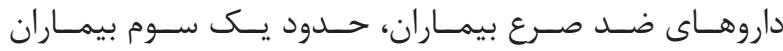

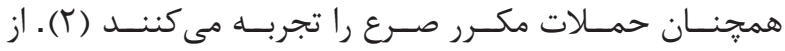

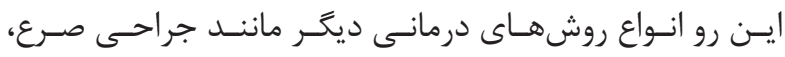

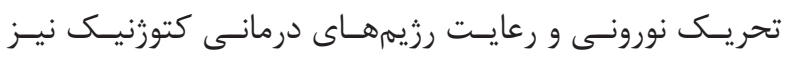

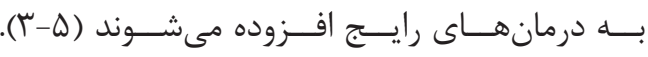

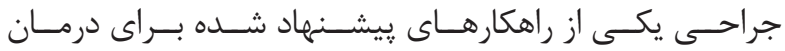

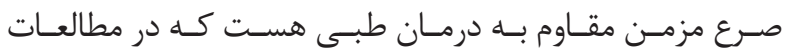

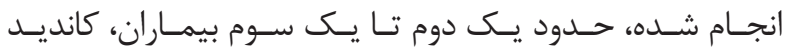

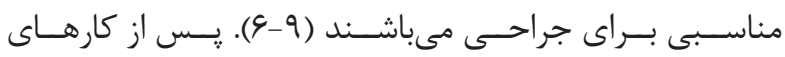

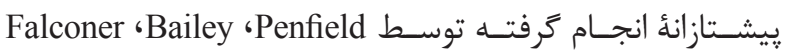

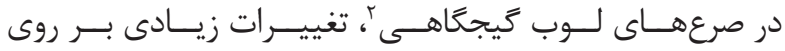

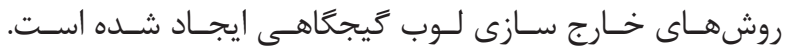

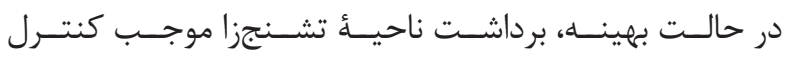

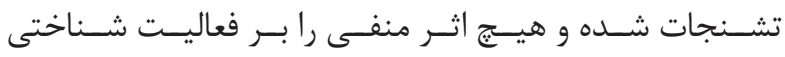

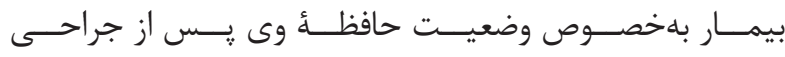

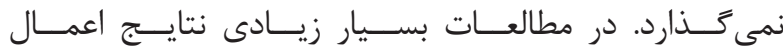

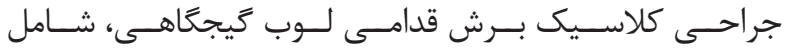

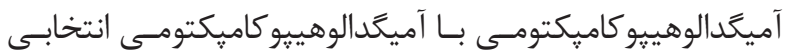

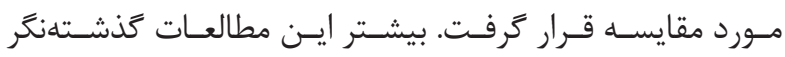

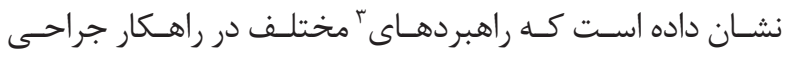

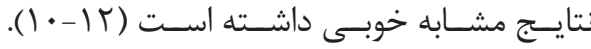

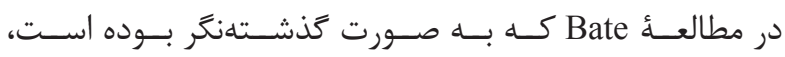

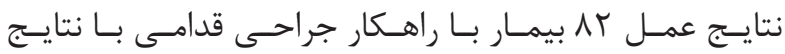

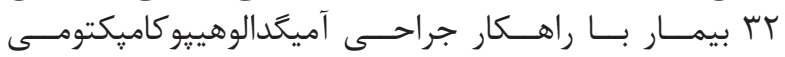

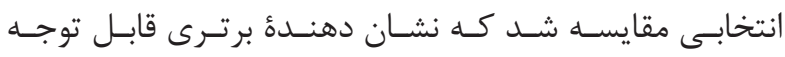

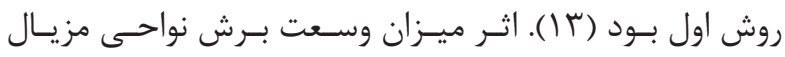

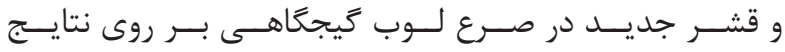

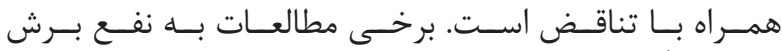

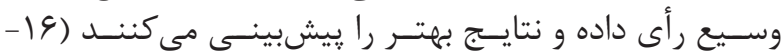

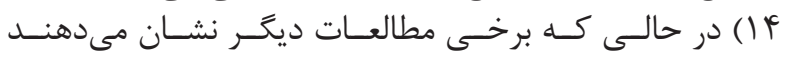

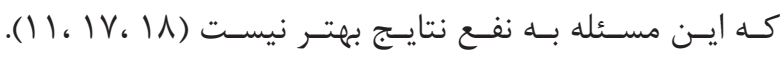

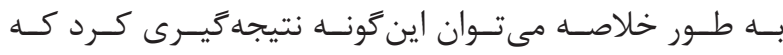

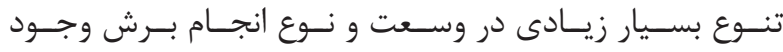

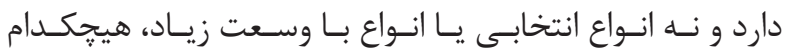

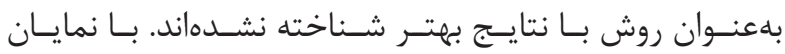

${ }^{1}$ World health organization

${ }^{2}$ Temporal lobe

${ }^{3}$ Strategies 


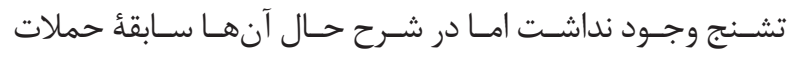

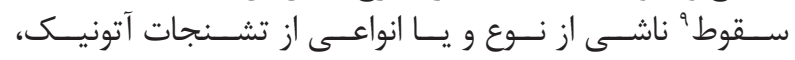

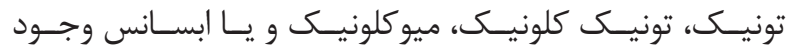

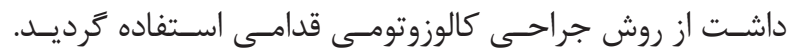

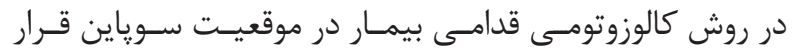

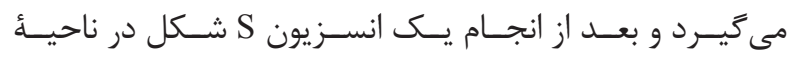

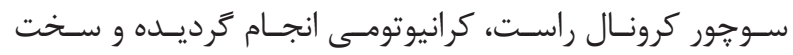

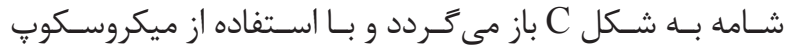

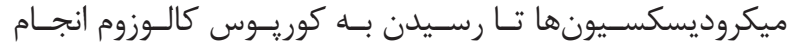

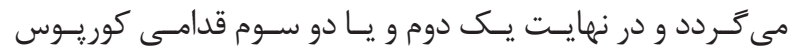

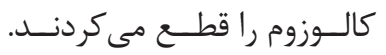

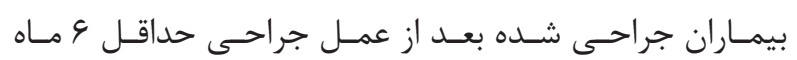

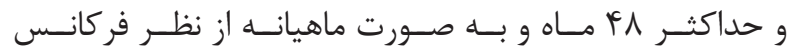

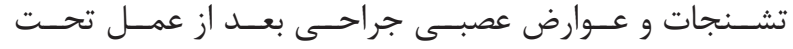

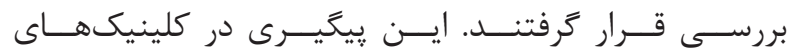

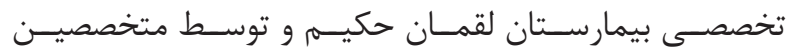

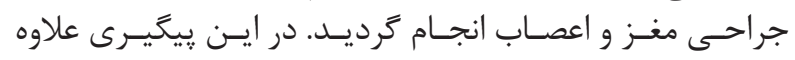

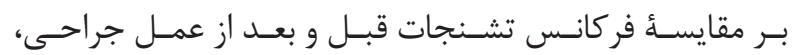

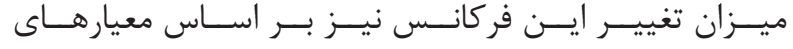
Engel

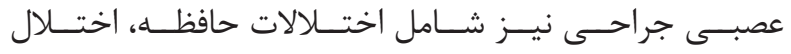

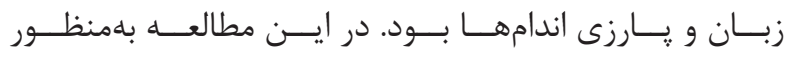

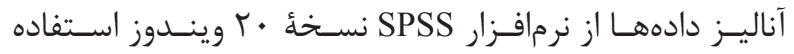

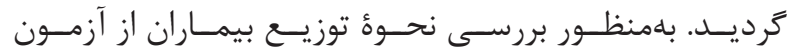

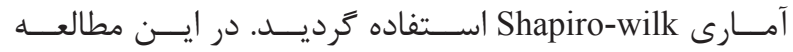

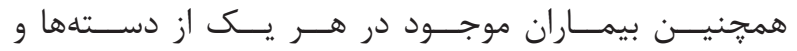

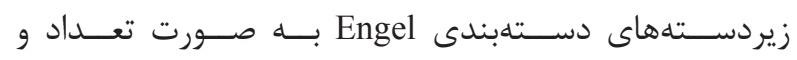

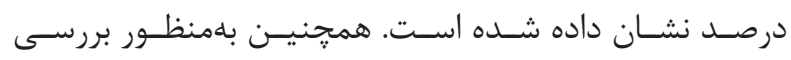

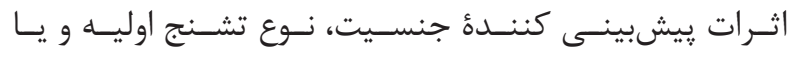

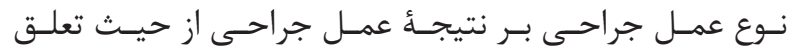

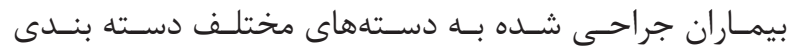

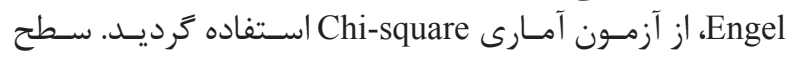

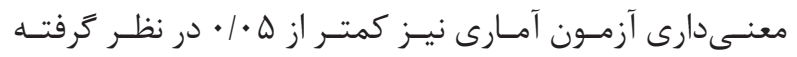

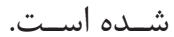

يافته ها

مشخصات جمعيت مورد مطالعه

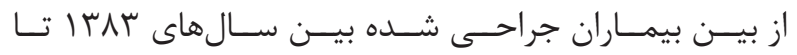

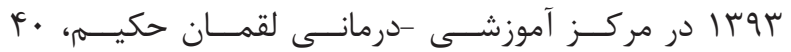

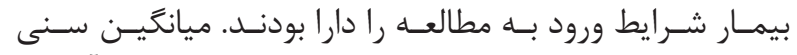

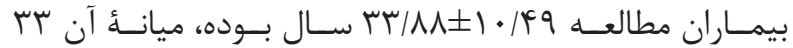

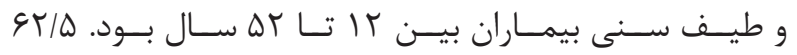

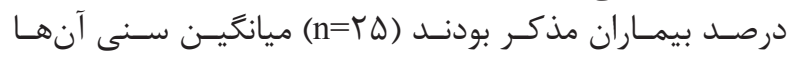

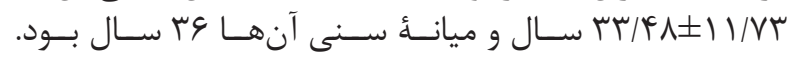

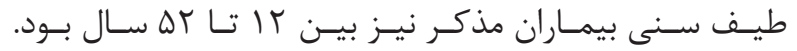

${ }^{4}$ Magnetic resonance imaging

${ }^{5}$ Electroencephalography

${ }^{6}$ Single-photon emission computed tomography
انجحامــــده باشـــند.

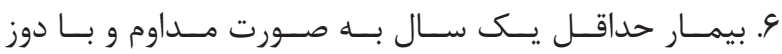

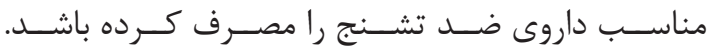

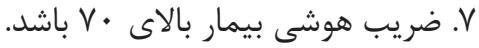

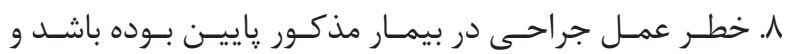

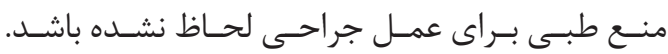

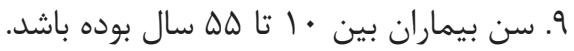

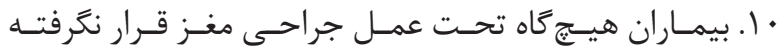

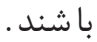

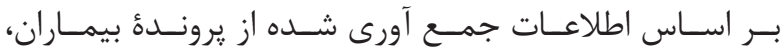

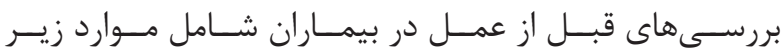
بـوده اســت ا. شرح حال دقيق بيماران و معاينات بالينى.

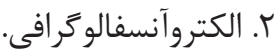

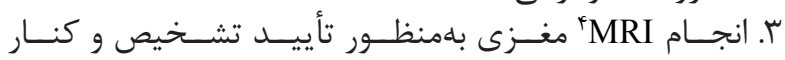
كذاشــتن ســاير علتهــــا. F. بررسى IQ بيمار و شرايط سير عصبى -روانى.

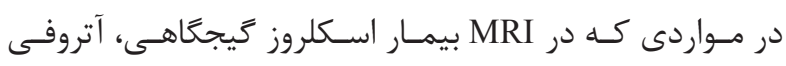

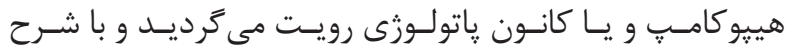

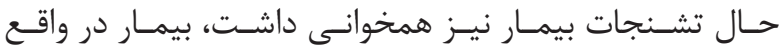

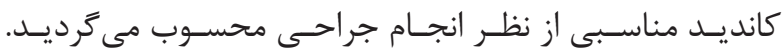

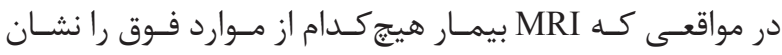

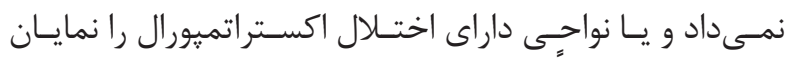

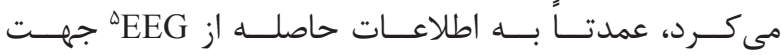

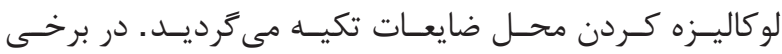

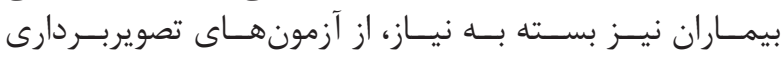

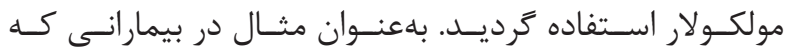

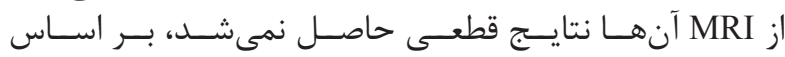

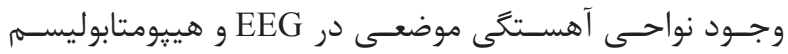

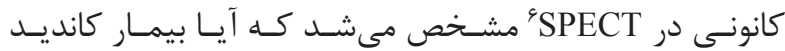

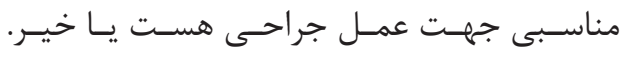

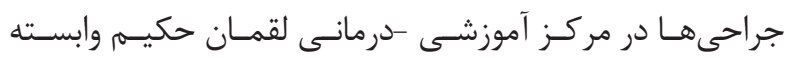

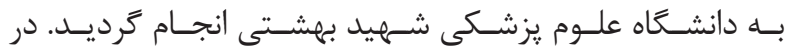

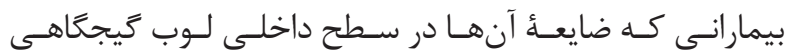

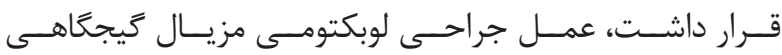

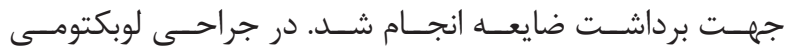

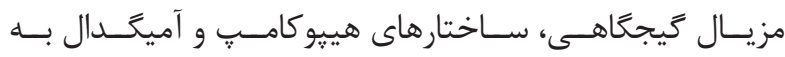

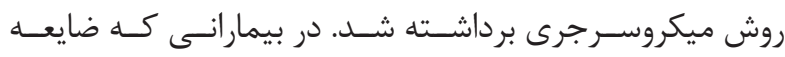

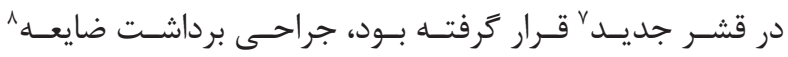

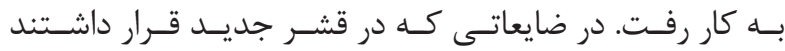

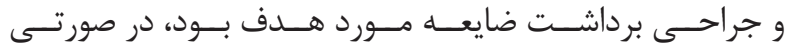

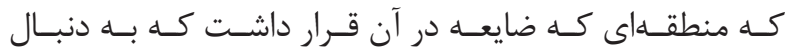

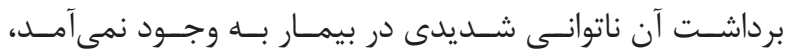

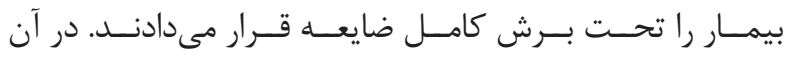

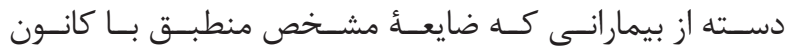

\footnotetext{
${ }^{7}$ Neocortex

${ }^{8}$ Lesionectomy

${ }^{9}$ Drop attack
} 


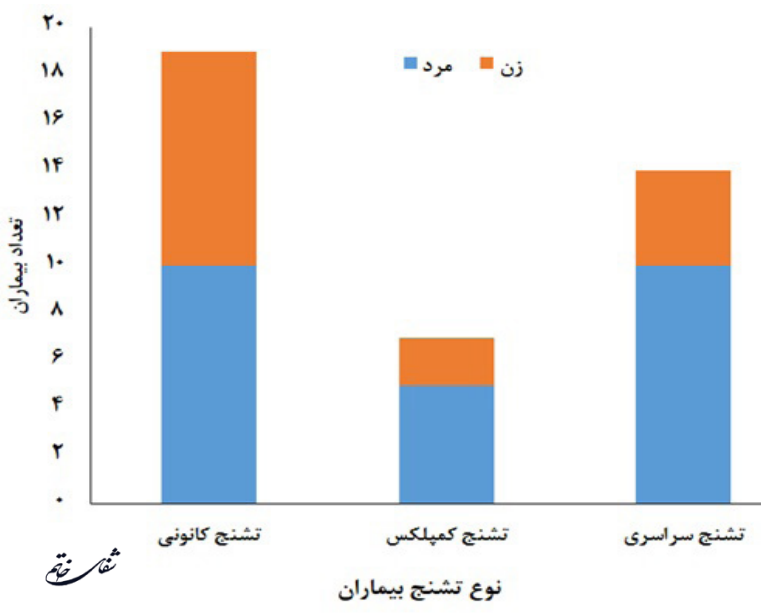

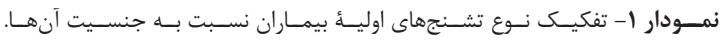

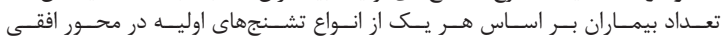

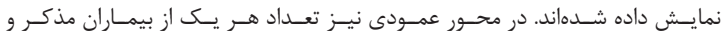

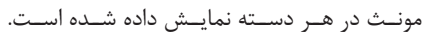

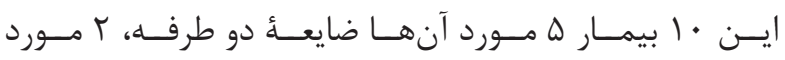

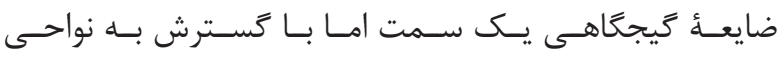

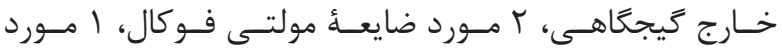

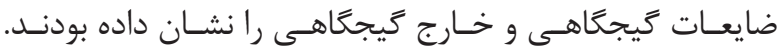

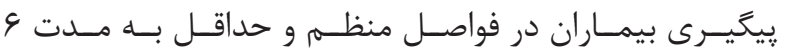

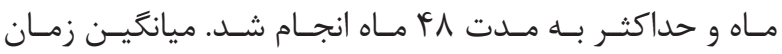

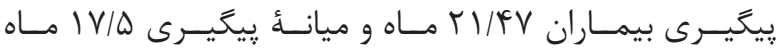

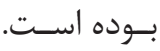

نتايج جراحى ها با توجه به جنسيت بيماران

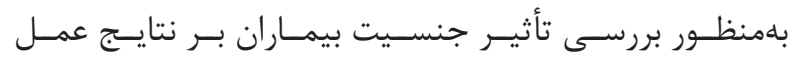

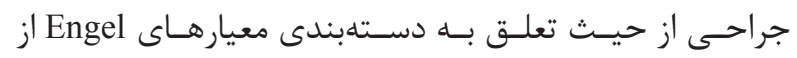

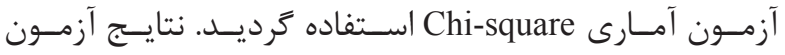

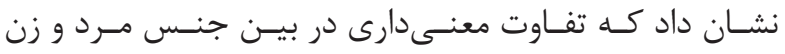

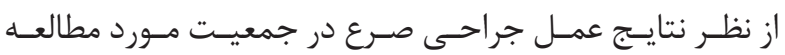

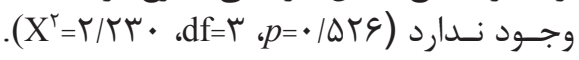

نتايج جراحى ها با توجه به نوع تشنج اوليه

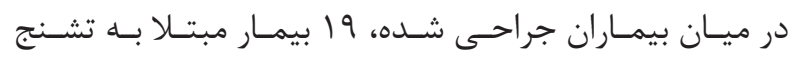

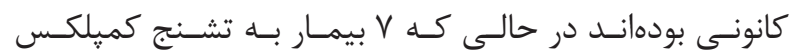

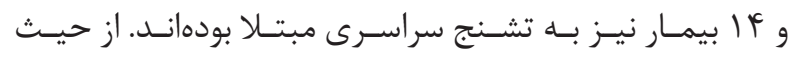

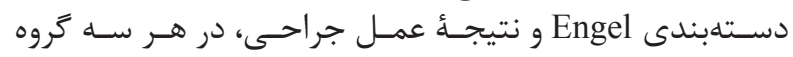

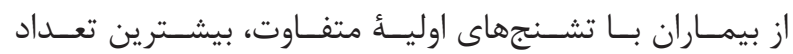

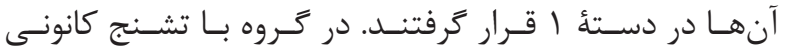

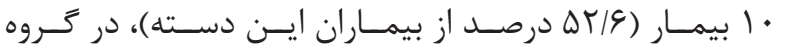

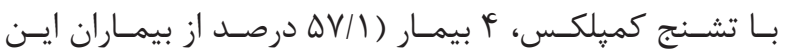

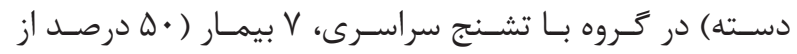

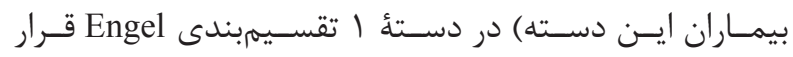

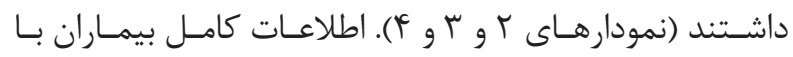

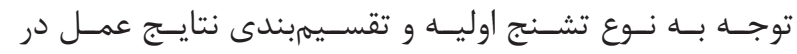

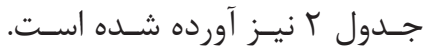

${ }^{10}$ Generalized seizure

${ }^{11}$ Complex-partial seizure

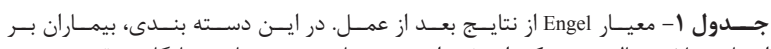

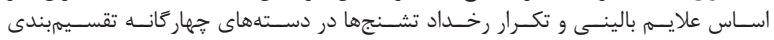

\begin{tabular}{|c|}
\hline كلاس ا: بدون تشنجهاى ناتوان كنيده \\
\hline 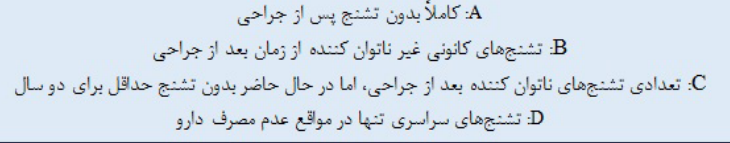 \\
\hline كلاس r: تشنجماى ناتوان كننده بهندرت \\
\hline 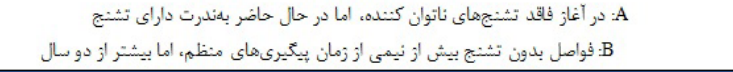 \\
\hline كلاس r: بهبود ارزشمند علايم \\
\hline 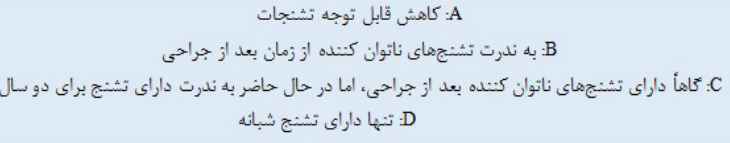 \\
\hline كلاس f: فاقد بهبود ارزشمند علايم \\
\hline 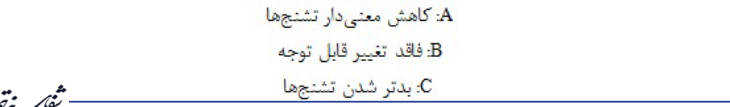 \\
\hline
\end{tabular}

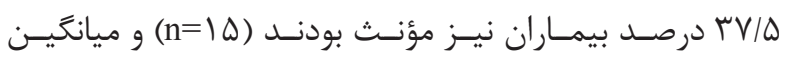

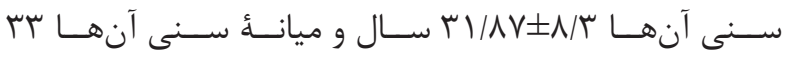

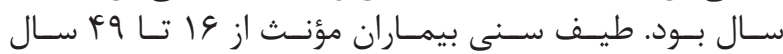

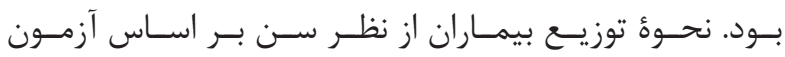

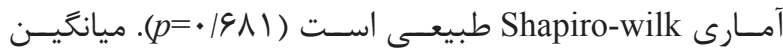

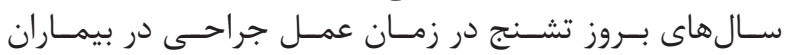

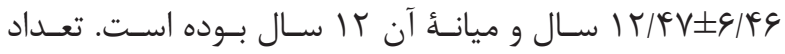

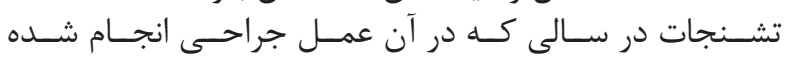

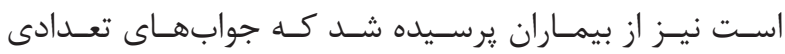

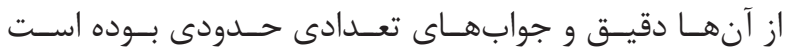

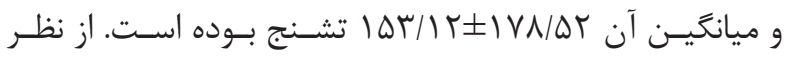

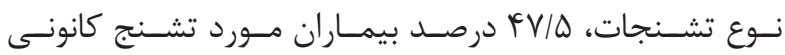

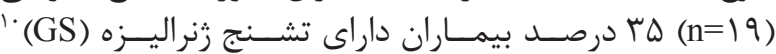

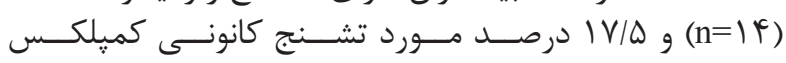

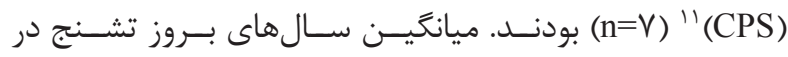

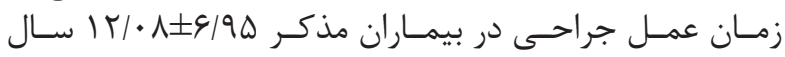

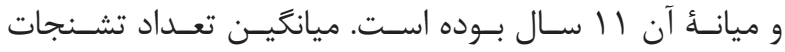

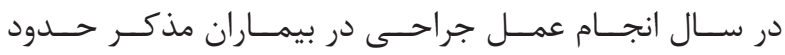

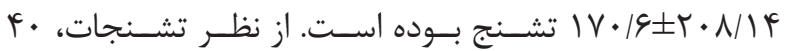

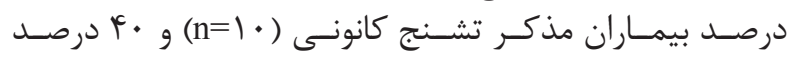

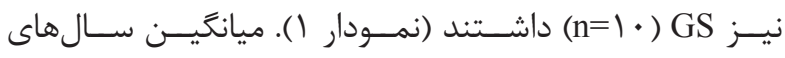

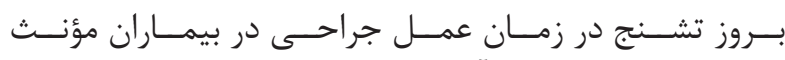
كا

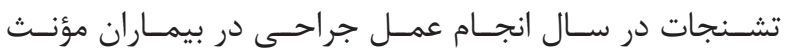

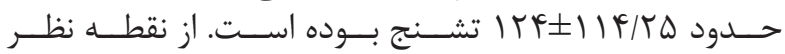

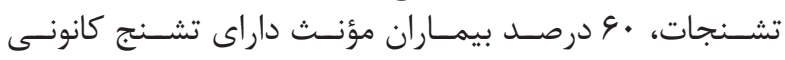

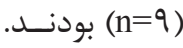

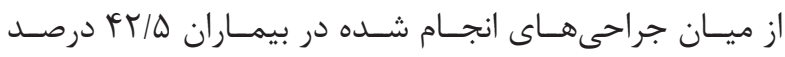

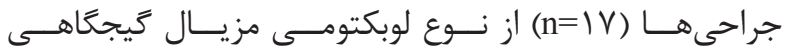

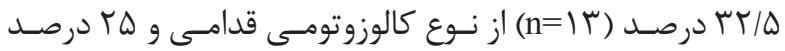

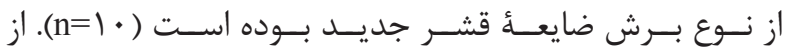




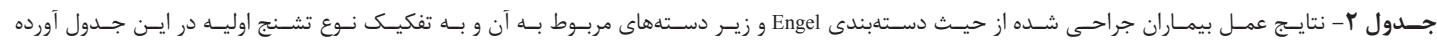

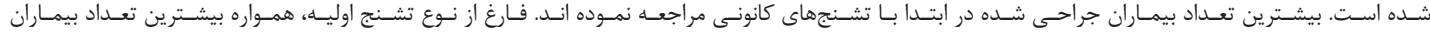

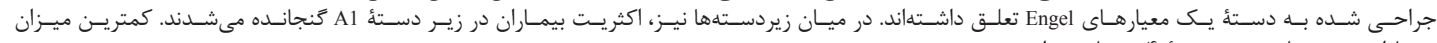

\begin{tabular}{|c|c|c|c|c|c|c|c|c|c|c|c|c|c|c|c|}
\hline \multicolumn{10}{|c|}{ تعداد (درصد) در هر حسته } & \multicolumn{4}{|c|}{ تعداد (درصد) در هر ستحه } & تعلداد & تشتع \\
\hline$F \mathbf{B}$ & $F A$ & rA & TD & TC & TB & TA & ID & IC & IA & F & $r$ & t & , & & عسته/ \\
\hline $\begin{array}{c}1 \\
(\Delta / T)\end{array}$ & - & $\begin{array}{c}r \\
(1 \Delta / \Lambda)\end{array}$ & - & 1 & $\begin{array}{c}1 \\
(\Delta / T)\end{array}$ & $\begin{array}{c}r \\
(10 / \mu)\end{array}$ & v & $\begin{array}{c}\Delta \\
(T / T)\end{array}$ & $\begin{array}{c}\Delta \\
\Delta \\
(T S / T)\end{array}$ & $\begin{array}{c}1 \\
(\Delta / r)\end{array}$ & $\begin{array}{c}r \\
(1 \otimes / A)\end{array}$ & $\begin{array}{c}\Delta \\
0 \\
(\tau g / T)\end{array}$ & $\begin{array}{c}1- \\
(\Delta T / \Omega)\end{array}$ & 19 & كاتوتئج \\
\hline r & $\begin{array}{c}1 \\
(1+/ 4)\end{array}$ & 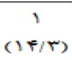 & - & - & $\begin{array}{c}1 \\
(1 \% / T)\end{array}$ & * & . & (1) & $\begin{array}{c}r \\
(F+/ 9)\end{array}$ & $\begin{array}{c}1 \\
(1+/ T)\end{array}$ & $\begin{array}{c}1 \\
(1+2 / m)\end{array}$ & $\begin{array}{c}1 \\
(1+/ T)\end{array}$ & $\begin{array}{c}F \\
(\Delta \vee / 1)\end{array}$ & $r$ & كميلكسي \\
\hline - & (vil) & $\begin{array}{c}T \\
(1+/ 4)\end{array}$ & $\begin{array}{c}1 \\
(\mathrm{v} / 1)\end{array}$ & (i) & . & $\begin{array}{c} \\
(1+\pi / m)\end{array}$ & (iil) & $\begin{array}{c}r \\
(1 / F / r)\end{array}$ & $\begin{array}{c}F \\
\left(\text { (TN/) }^{2}\right.\end{array}$ & (viv) & $\begin{array}{c}T^{\prime} \\
(1 F / M)\end{array}$ & $\begin{array}{c}F \\
\left(T^{\prime} /(s)\right.\end{array}$ & $\begin{array}{c}r \\
(\Delta-)\end{array}$ & if & سراسترى \\
\hline $\begin{array}{c}1 \\
(T / \theta)\end{array}$ & $\begin{array}{l}r \\
\text { (s) }\end{array}$ & $\begin{array}{c}\Rightarrow \\
(10)\end{array}$ & $\begin{array}{c}1 \\
(T / \theta)\end{array}$ & $\begin{array}{c}T \\
(\Delta)\end{array}$ & $\begin{array}{c}T \\
(\Delta)\end{array}$ & $\begin{array}{c}\Delta \\
(i r / \Delta)\end{array}$ & $\begin{array}{c}1 \\
(\pi / \theta)\end{array}$ & $\hat{(r-)}$ & $\begin{array}{c}\text { Ir } \\
(r \cdot)\end{array}$ & $\begin{array}{c}r \\
(v / \Delta)\end{array}$ & $\begin{array}{c}8 \\
(10)\end{array}$ & $\begin{array}{c}1 \cdot \\
(T \Delta)\end{array}$ & $\begin{array}{c}r y \\
(\Delta T / \Delta)\end{array}$ & $F$ & موع \\
\hline
\end{tabular}

يكسى از مشــاهدات ايـن مطالعـه توزيــع بيشـتر بيمـاران در

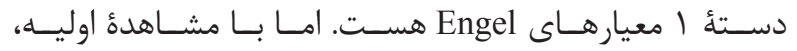

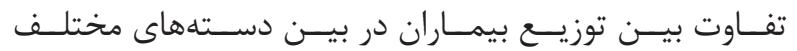

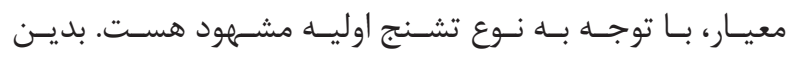

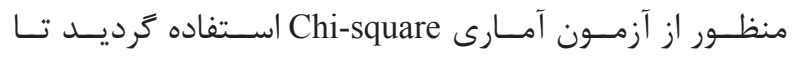

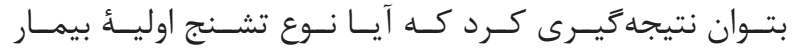

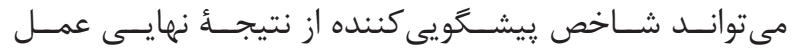

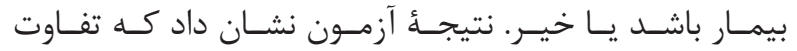

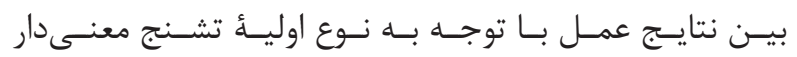
نيسـت (X)

$$
\text { نتايج به تفكيك نوع عمل جراحى }
$$

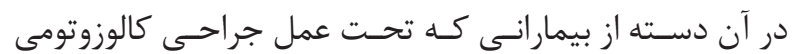

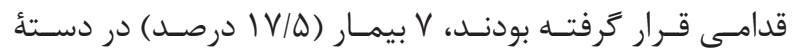

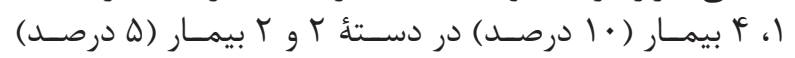

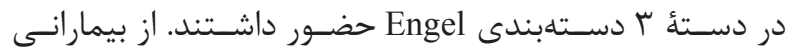

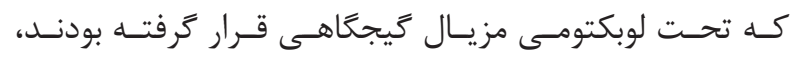

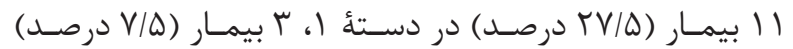

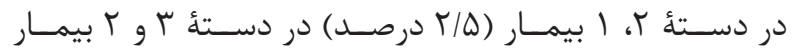

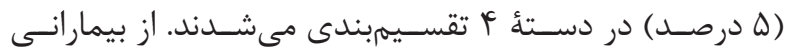

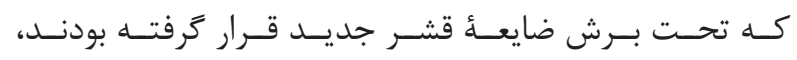

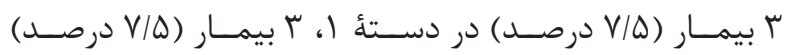

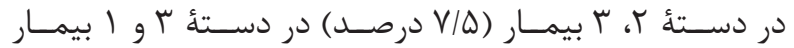

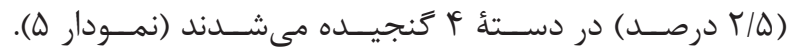

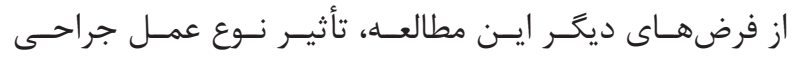

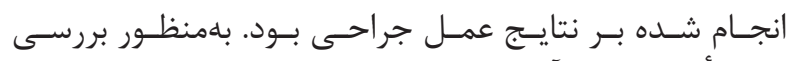

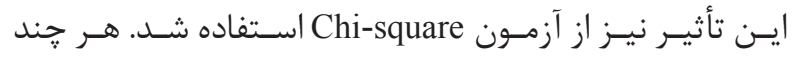

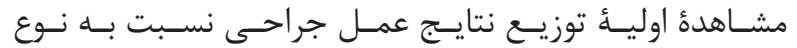

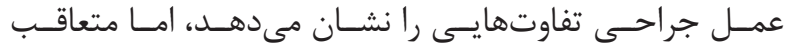

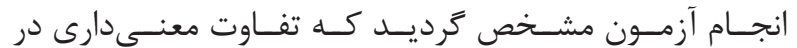

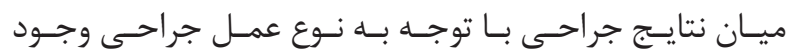

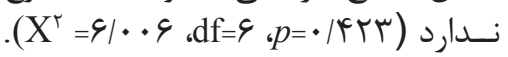

$$
\text { عوارض جراحىها }
$$

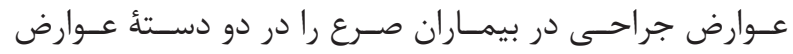

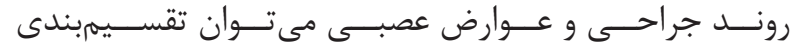

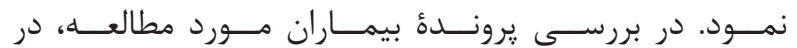

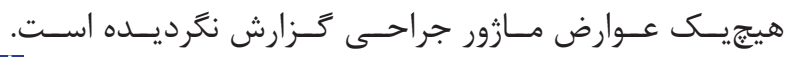
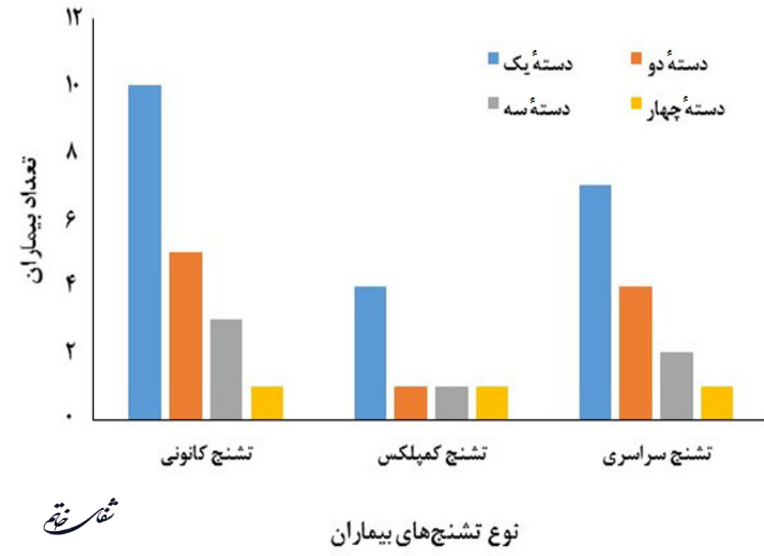

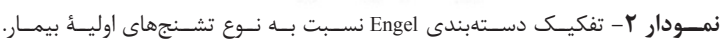

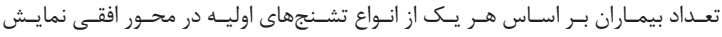

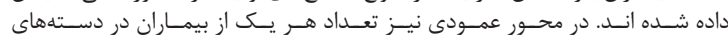

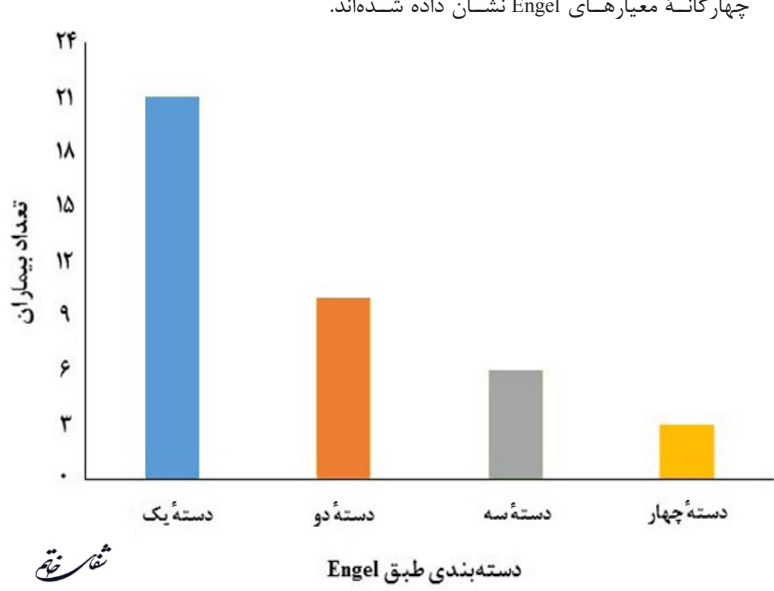

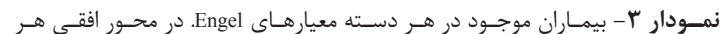

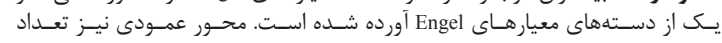

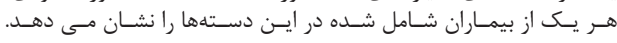

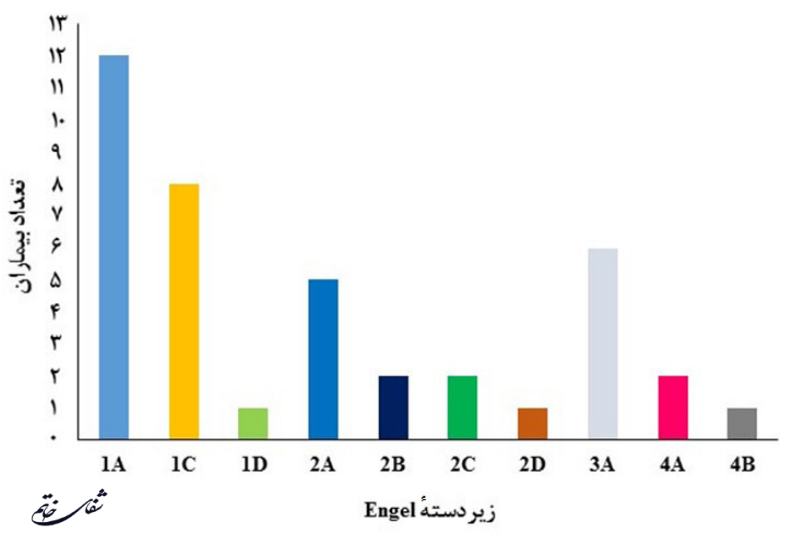

نمودار \$- بيماران موجود در هر زير دسته معيارهاى زيت Engel 


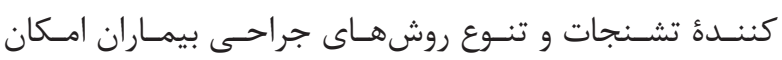

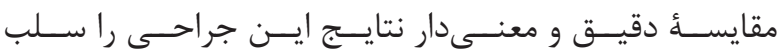

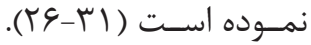

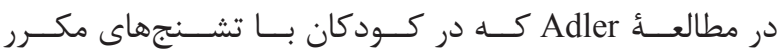

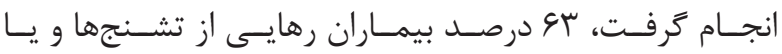

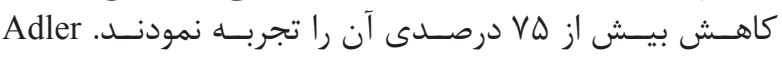

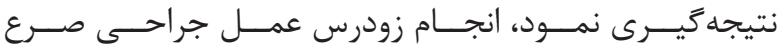

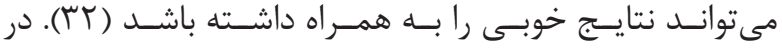

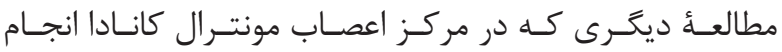

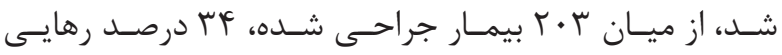

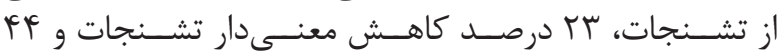

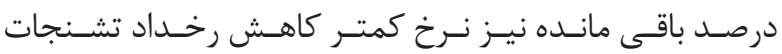

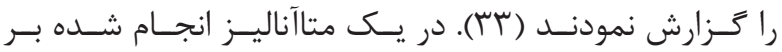

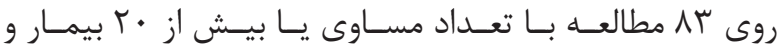

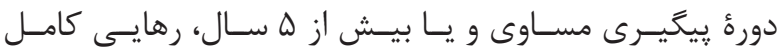

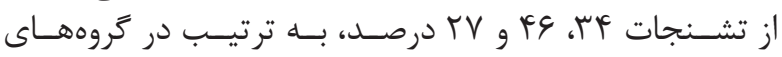

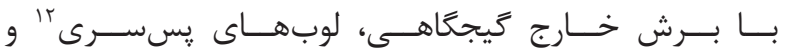

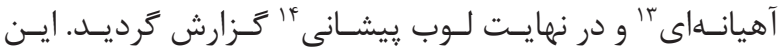

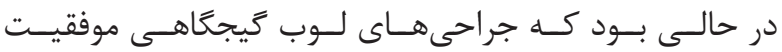

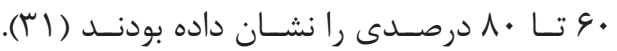

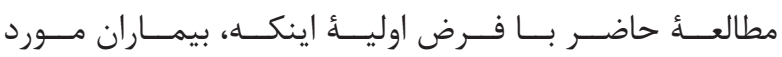

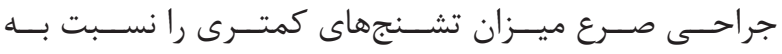

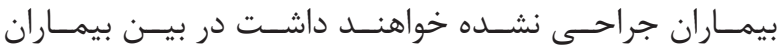

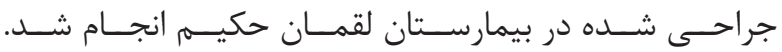

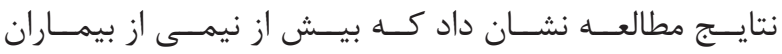

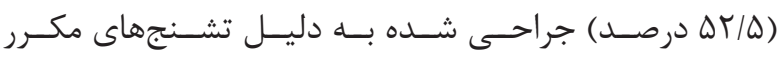

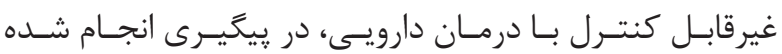

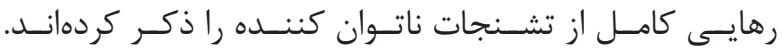

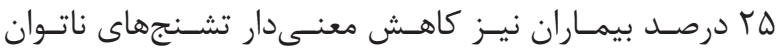

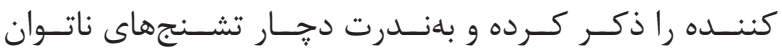

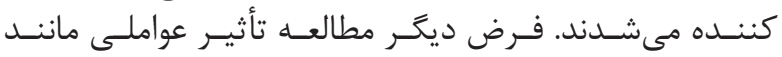

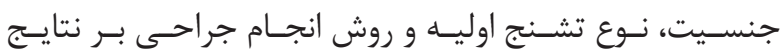

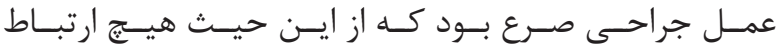

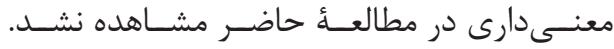

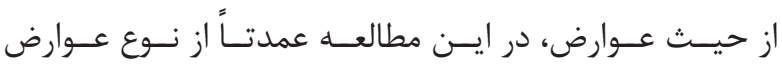

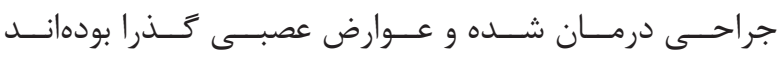

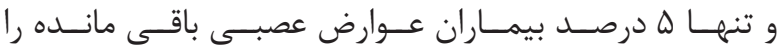

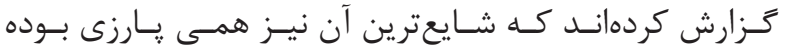

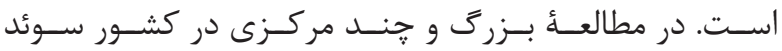

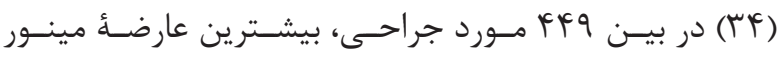

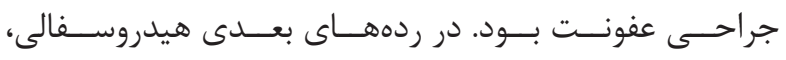

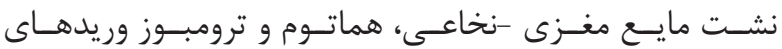

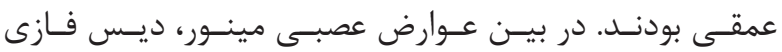

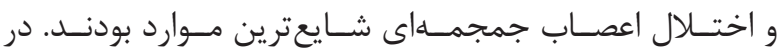

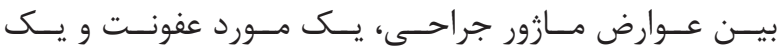

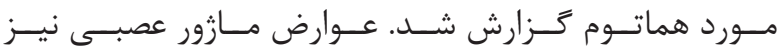

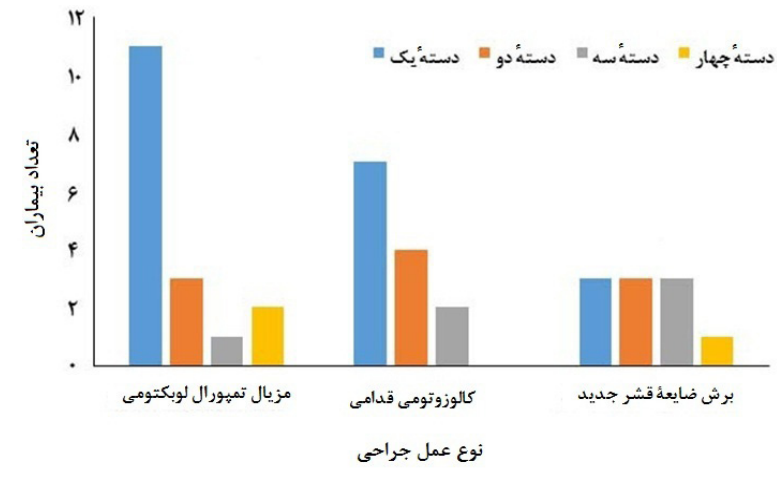

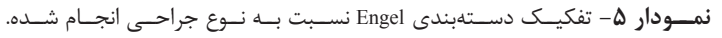

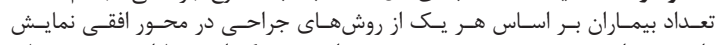

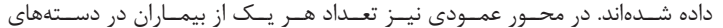

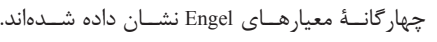

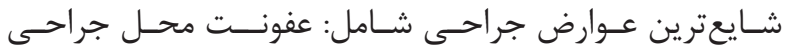

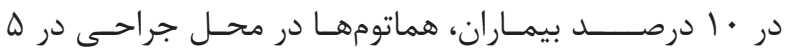

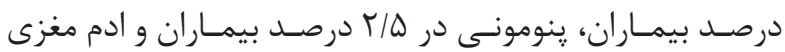

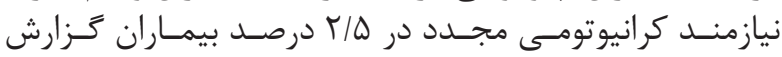

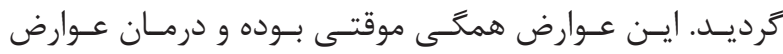

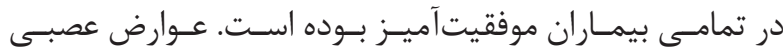

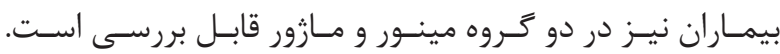

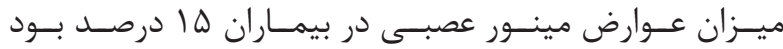

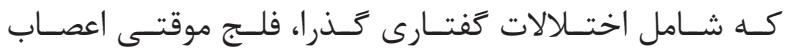

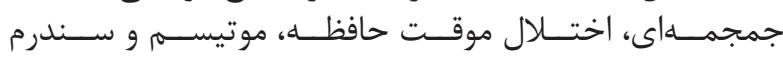

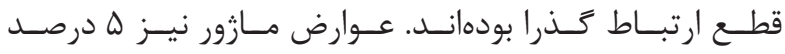

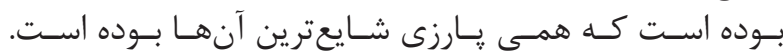
بحث و نتيجه كيرى

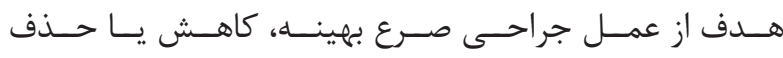

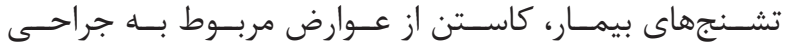

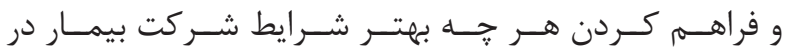

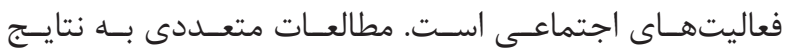

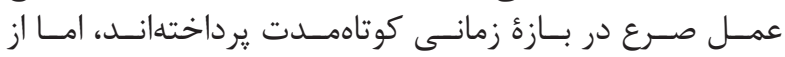

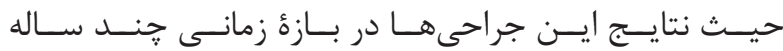

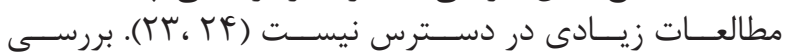

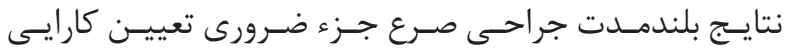

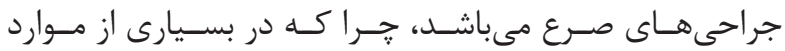

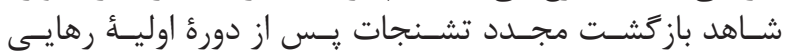

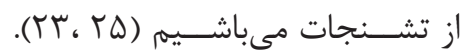

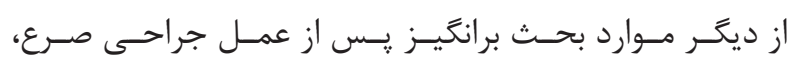

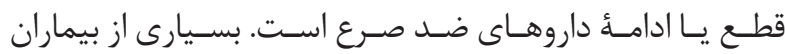

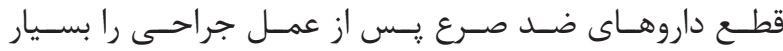

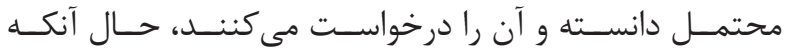

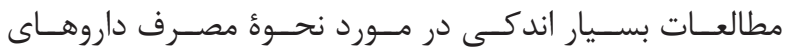

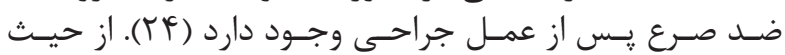

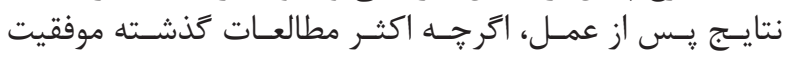

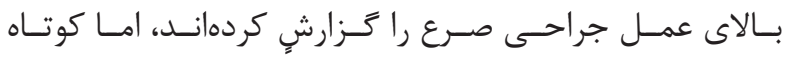

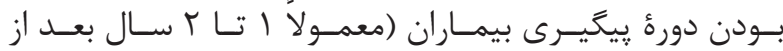

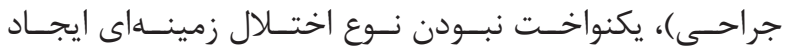

\footnotetext{
${ }^{12}$ Occipital lob

${ }^{13}$ Parietal lob

${ }^{14}$ Frontal lob
} 


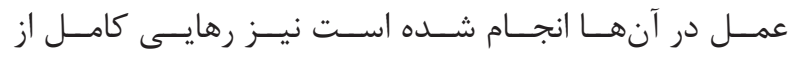

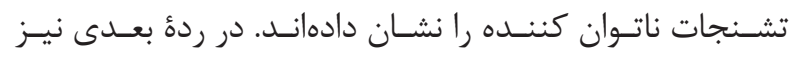

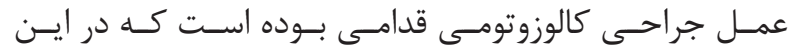

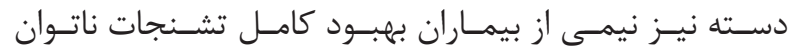

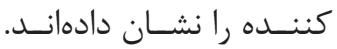

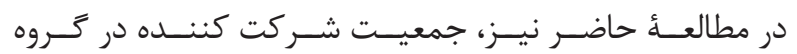

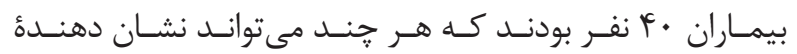

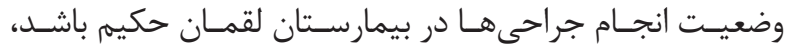

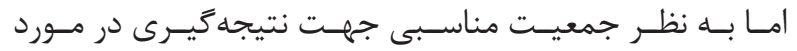

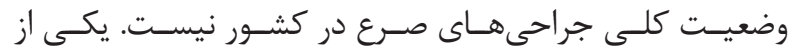

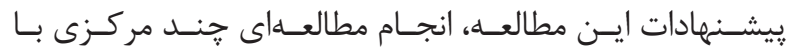

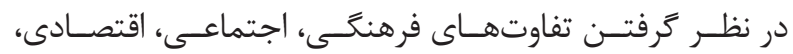

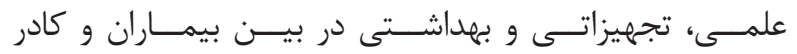

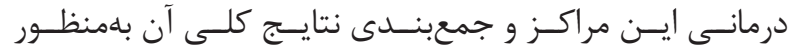

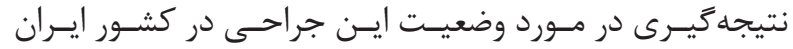

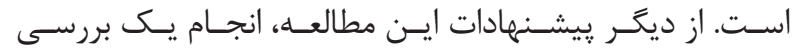

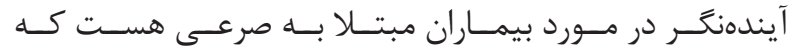

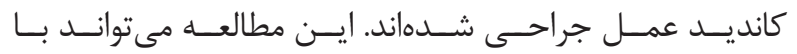

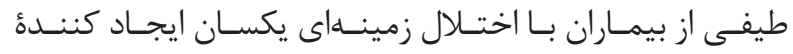

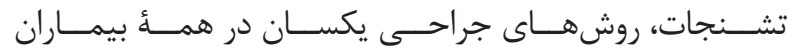

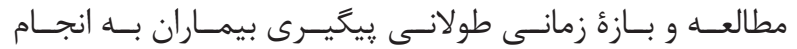

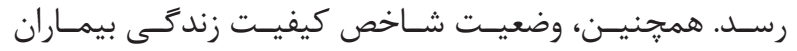

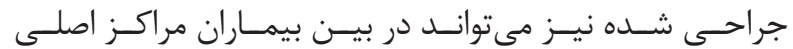

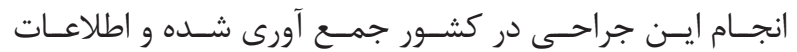

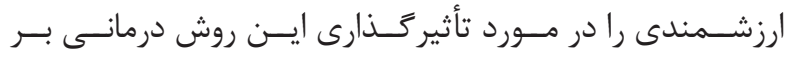

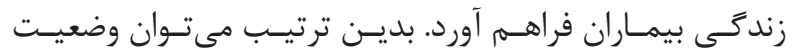

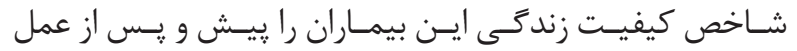

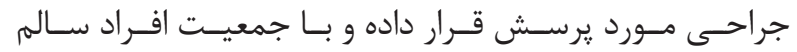

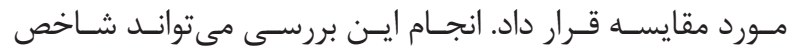

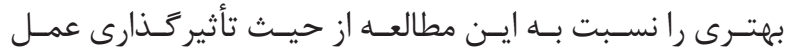

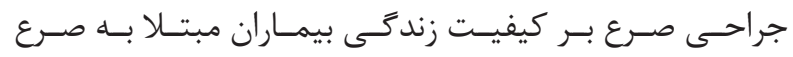

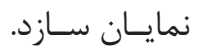

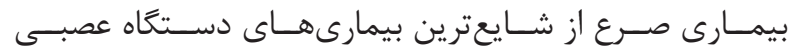

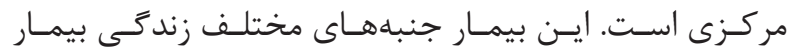

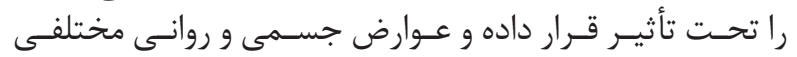

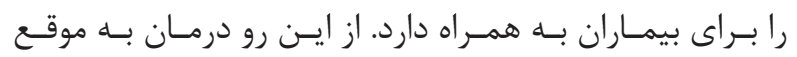

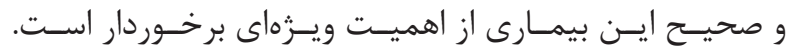

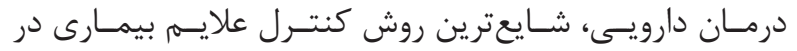

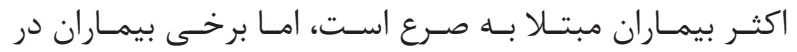

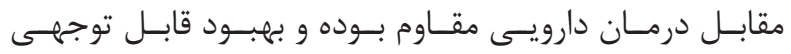

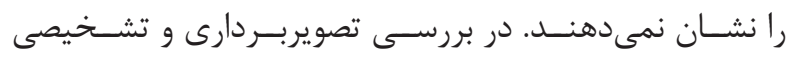

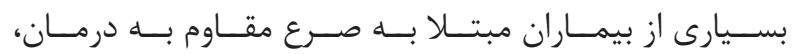

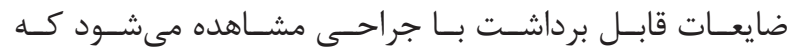

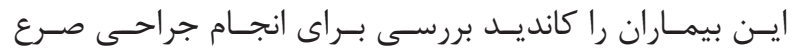

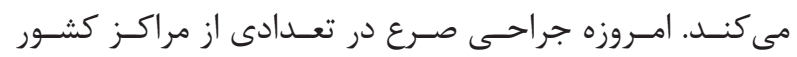

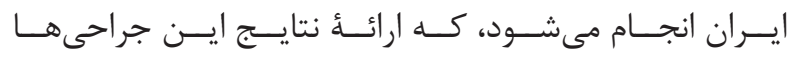

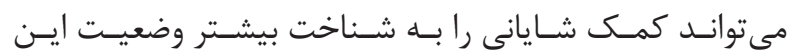

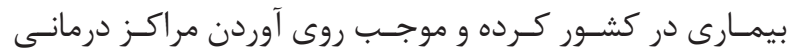

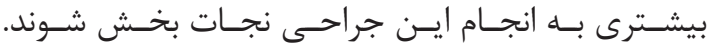

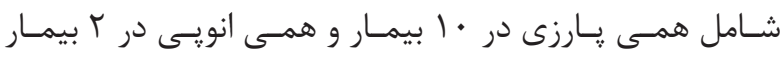

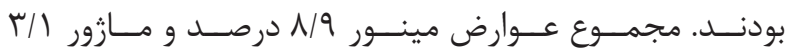

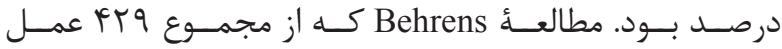

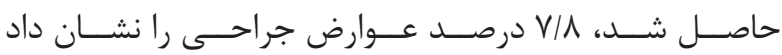

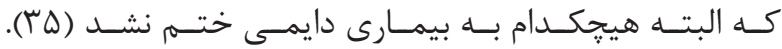

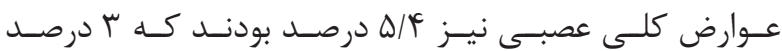

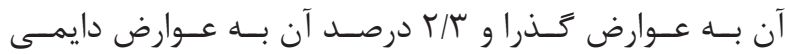

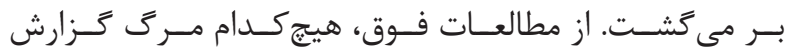

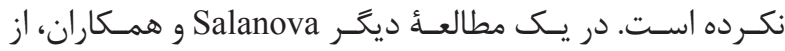

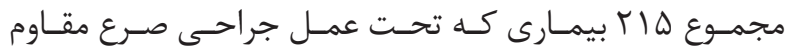

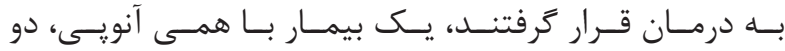

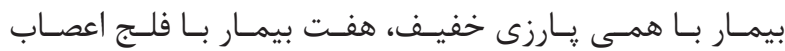

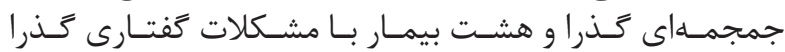

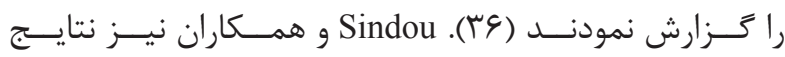

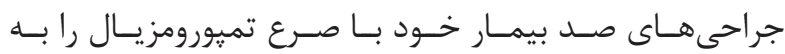

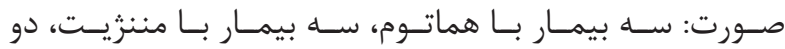

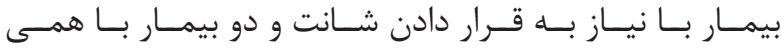

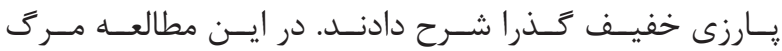

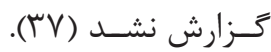

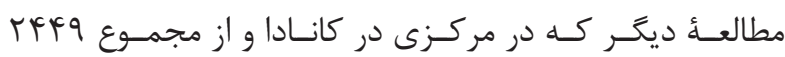

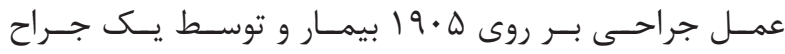

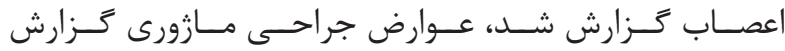

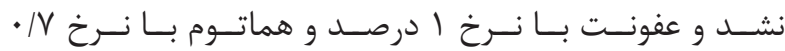

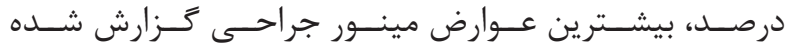

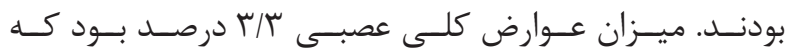

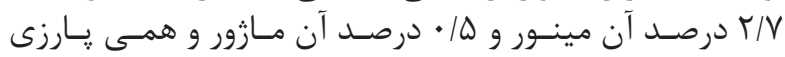

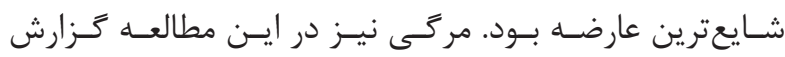

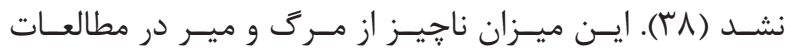

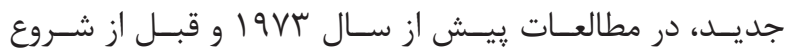

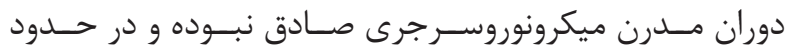

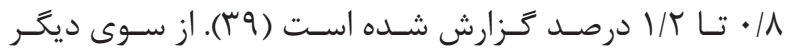

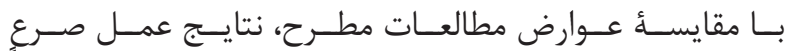

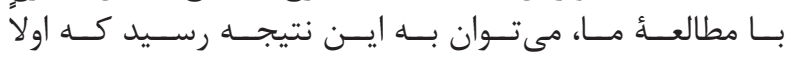

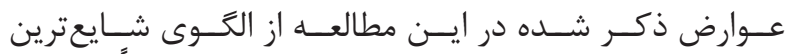

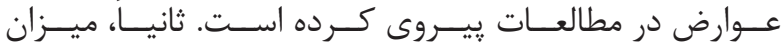

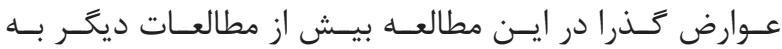

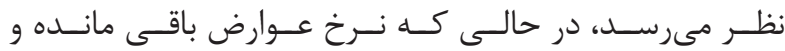

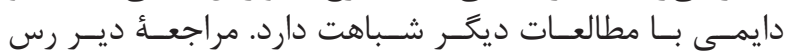

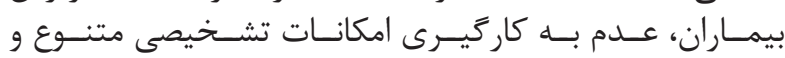

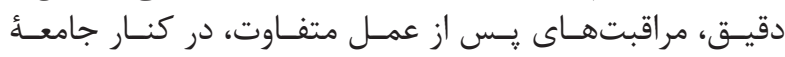

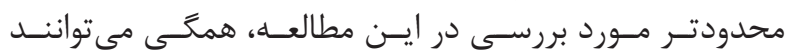

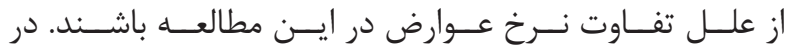

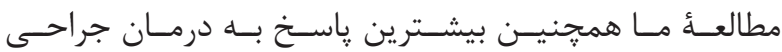

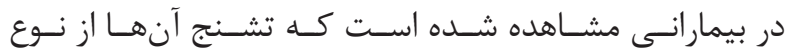

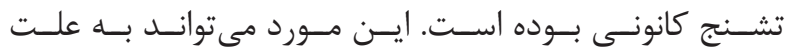

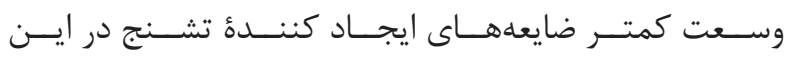

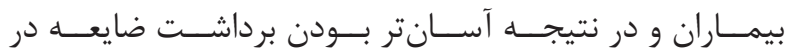

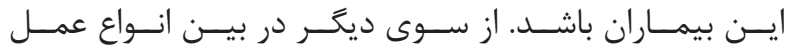

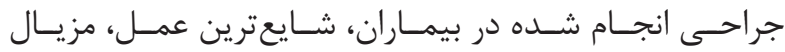

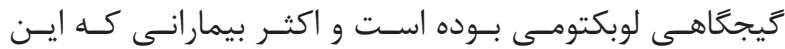


1. Ball JR, Hurlbert RJ, Winn HR. YOUMANS Neurological Surgery. 6th. Philadelphia. 2011.

2. Brodie M, Barry S, Bamagous G, Norrie J, Kwan P. Patterns of treatment response in newly diagnosed epilepsy. Neurology. 2012; 78(20): 1548-54.

3. Ahmadi M, Sharifi MS. Treatments of Parkinson's disease, Epilepsy and obsessive compulsive disorder with deep brain stimulation. Shefaye Khatam. 2014; 2(1): $95-100$.

4. Lefevre F, Aronson N. Ketogenic diet for the treatment of refractory epilepsy in children: a systematic review of efficacy. Pediatrics. 2000; 105(4): e46.

5. Boon P, Vonck K, De Herdt V, Van Dycke A, Goethals $\mathrm{M}$, Goossens L, et al. Deep brain stimulation in patients with refractory temporal lobe epilepsy. Epilepsia. 2007; 48(8): 1551-60.

6. Keogan M, McMackin D, Peng S, Phillips J, Burke $\mathrm{T}$, Murphy S, et al. Temporal neocorticectomy in management of intractable epilepsy: long-term outcome and predictive factors. Epilepsia. 1992; 33(5): 852-61.

7. Gates JR, Leppik IE, Yap J, Gumnit RJ. Corpus callosotomy: clinical and electroencephalographic effects. Epilepsia. 1984; 25(3): 308-16.

8. Engel Jr J. Surgery for seizures. N Engl J Med. 1996; 334(10): 647-53.

9. Spencer S, Spencer D, Williamson P, Sass K, Novelly R, Mattson R. Corpus callosotomy for epilepsy. I. seizure effects. Neurology. 1988; 38(1): 19-24.

10. Clusmann H, Schramm J, Kral T, Helmstaedter C, Ostertun B, Fimmers R, et al. Prognostic factors and outcome after different types of resection for temporal lobe epilepsy. J Neurosurg. 2002; 97(5): 1131-41.

11. Arruda F, Cendes F, Andermann F, Dubeau F, Villemure J, Jones-Gotman M, et al. Mesial atrophy and outcome after amygdalohippocampectomy or temporal lobe removal. Ann Neurol. 1996; 40(3): 446-50.

12. Paglioli E, Palmini A, Portuguez M, Paglioli E, Azambuja N, Costa JCd, et al. Seizure and memory outcome following temporal lobe surgery: selective compared with nonselective approaches for hippocampal sclerosis. J Neurosurg. 2006; 104(1): 70-8.

13. Bate H, Eldridge P, Varma T, Wieshmann U. The seizure outcome after amygdalohippocampectomy and temporal lobectomy. Eur J Neurol. 2007; 14(1): 90-4.
14. Wyler AR, Hermann BP, Somes G. Extent of medial temporal resection on outcome from anterior temporal lobectomy: a randomized prospective study. Neurosurgery. 1995; 37(5): 982-91.

15. Bonilha L, Yasuda CL, Rorden C, Li LM, Tedeschi $\mathrm{H}$, De Oliveira E, et al. Does resection of the medial temporal lobe improve the outcome of temporal lobe epilepsy surgery? Epilepsia. 2007; 48(3): 571-8.

16. Shamim S, Wiggs E, Heiss J, Sato S, Liew C, Solomon J, et al. Temporal lobectomy: resection volume, neuropsychological effects, and seizure outcome. Epilepsy Behav. 2009; 16(2): 311-4.

17. Wolf RL, Ivnik RJ, Hirschorn KA, Sharbrough FW, Cascino GD, Marsh WR. Neurocognitive efficiency following left temporal lobectomy: standard versus limited resection. J Neurosurg. 1993; 79(1): 76-83.

18. Van Rijckevorsel K, Grandin C, De Tourtchaninoff M, Vaz G, Raftopoulos C. Selective amygdalohippocampectomy: seizure outcome in 26 consecutive cases compared to the amount of resection. Epilepsia. 2005; 46: 253-60.

19. Wieser HG, Silfvenius H. Overview: Epilepsy Surgery in Developing Countries. Epilepsia. 2000; 41(4): S3-S9.

20. Sirven JI, Pedley TA, Wilterdink J. Evaluation and management of drug-resistant epilepsy. UpToDate. 2011; 13(17): 13585.

21. Kwan P, Arzimanoglou A, Berg AT, Brodie MJ, Allen Hauser W, Mathern G, et al. Definition of drug resistant epilepsy: consensus proposal by the ad hoc task force of the ILAE commission on therapeutic strategies. Epilepsia. 2010; 51(6): 1069-77.

22. Engel Jr J. International league against Epilepsy (ILAE). a proposed diagnostic scheme for people with epileptic seizures and with epilepsy: report of the ILAE task force on classification and terminology. Epilepsia. 2001; 42(6): 796-803.

23. Yoon HH, Kwon H, Mattson R, Spencer D, Spencer S. Long-term seizure outcome in patients initially seizure-free after resective epilepsy surgery. Neurology. 2003; 61(4): 445-50.

24. McIntosh AM, Kalnins RM, Mitchell LA, Fabinyi GC, Briellmann RS, Berkovic SF. Temporal lobectomy: long-term seizure outcome, late recurrence and risks for seizure recurrence. Brain. 2004; 127(9): 2018-30. 
25. Wingkun EC, Awad IA, Lüders H, Awad CA. Natural history of recurrent seizures after resective surgery for epilepsy. Epilepsia. 1991; 32(6): 851-6.

26. Cohen-Gadol AA, Wilhelmi BG, Collignon F, White JB, Britton JW, Cambier DM, et al. Long-term outcome of epilepsy surgery among 399 patients with nonlesional seizure foci including mesial temporal lobe sclerosis. J Neurosurg. 2006; 104(4): 513-24.

27. Guldvog B, LøSyning Y, Hauglie-Hanssen E, Flood S, BjøSrnaes H. Surgical versus medical treatment for epilepsy II. outcome related to social areas. Epilepsia. 1991; 32(4): 477-86.

28. Lieb JP, Engel J, Babb TL. Interhemispheric propagation time of human hippocampal seizures. Epilepsia. 1986; 27(3): 286-93.

29. McIntosh A, Wilson SJ, Berkovic SF. Seizure outcome after temporal lobectomy: current research practice and findings. Epilepsia. 2001; 42(10): 1288307.

30. Spencer SS. Long-term outcome after epilepsy surgery. Epilepsia. 1996; 37(9): 807-13.

31. Téllez-Zenteno JF, Dhar R, Wiebe S. Longterm seizure outcomes following epilepsy surgery: a systematic review and meta-analysis. Brain. 2005; 128(5): 1188-98.

32. Adler J, Erba G, Winston KR, Welch K, Lombroso CT. Results of surgery for extratemporal partial epilepsy that began in childhood. Arch Neurol. 1991;
48(2): $133-40$.

33. Rasmussen T. Surgery for central, parietal and occipital epilepsy. Can J Neurol Sci. 1991; 18(S4): 611-6.

34. Rydenhag B, Hans C. Complications of epilepsy surgery after 654 procedures in Sweden, september 1990-1995: a multicenter study based on the swedish national epilepsy surgery register. Neurosurgery. 2001; 49(1): 51-7.

35. Behrens E, Schramm J, Zentner J, König R. Surgical and neurological complications in a series of 708 epilepsy surgery procedures. Neurosurgery. 1997; 41(1): 1-10.

36. Salanova V, Markand O, Worth R. Temporal lobe epilepsy surgery: outcome, complications, and late mortality rate in 215 patients. Epilepsia. 2002; 43(2): 170-4.

37. Sindou M, Guenot M, Isnard J, Ryvlin P, Fischer C, Mauguiere F. Temporo-mesial epilepsy surgery: outcome and complications in 100 consecutive adult patients. Acta Neurochirurgica. 2006; 148(1): 39-45.

38. Tanriverdi T, Ajlan A, Poulin N, Olivier A. Morbidity in epilepsy surgery: an experience based on 2449 epilepsy surgery procedures from a single institution: clinical article. J Neurosurg. 2009; 110(6): 1111-23.

39. Jensen I. Temporal lobe surgery around the world results, complications, and mortality. Acta Neurol Scand. 1975; 52(5): 354-73. 